\title{
Failure to find spatial reversal deficits following medial frontal lesions
}

\author{
VENDER KNOWLES WEIR and ROGER K. THOMAS \\ University of Georgia, Athens, Georgia 30602
}

\begin{abstract}
Following training on four spatial reversals, rats were given lesions in the medial frontal (MF) frontopolar (FP), or parietal neocortex, or they received sham operations. None of the groups differed significantly postoperatively. This finding was contrary to an earlier report of deficits following MF lesions. The animals in that study received 64 or 104 (60 or 100 in a previous experiment) preoperative reversals. Their mean errors for four postoperative reversals were 6.3 and 5.2 , respectively. The MF group here had a mean of 5.2 errors for four postoperative reversals. Statistical comparison of the combined MF groups' data from the earlier study with our MF group data revealed no significant difference. However, the control groups from that study made significantly fewer errors than our controls postoperatively. It is suggested that spatial reversal deficits following MF lesions are subtle and may depend upon the extent of preoperative training received by control groups for their statistical confirmation.
\end{abstract}

The present experiment was one of two comparing the effects of lesions in the frontal and posterior association cortices in rats, and it was in this context that the rationale for the lesion sites and the task used here was developed. The report of an experiment which used Lashley's Maze III (Thomas \& Weir, in press) includes arguments pertinent to the existence and locations of frontal and posterior association cortices in rats. Therefore, these arguments are only summarized here.

Contrary to the traditional view that the frontal neocortical poles of the rat cerebrum may be the homolog of primate frontal association cortex, Leonard (1969) suggested cautiously that a medial frontal neocortical area and an area just dorsal to the rhinal sulcus may provide this homolog. The question of the existence and location of posterior association cortex in the rat was based, in part, on the classic works of Gurdjian (1927), Clark (1932), and Lashley (1941), together with consideration of Diamond and Hall's (1969) more recent work on the hedgehog and tree shrew and Thomas' (1970) reanalysis of the Lashley retention data.

Divac (1971) reported deficits in the retention of a spatial reversal problem following medial frontal, but not frontopolar neocortical lesions in the rat. On Lashley's Maze III, another problem with spatial components, medial frontal and parietal but not frontopolar lesions significantly impaired performance (Thomas \& Weir, in press).

Following training on four spatial reversals, rats received lesions in the medial frontal (MF), frontopolar

Derived from part of the MS thesis of the first author presented to the Graduate Faculty of the University of Georgia. This study, as part of the larger work, was presented at the 1973 meeting of the Southern Society for Philosophy and Psychology. Reprint requests should be addressed to Roger $K$. Thomas, Department of Psychology, University of Georgia, Athens, Georgia 30602.
(FP), or parietal neocortex, or they received sham operations. The rats were then given training on four postoperative reversals. It was expected that Divac's findings would be confirmed, and it was hoped that the extension of that study to include parietal lesions would be useful.

Our procedures essentially followed Divac's with one apparently critical exception. The rats in his experiment had been used in a prior study in which the animals were trained on either 60 or 100 reversals. We did not include these 60 or 100 reversals. Nevertheless, our findings were sufficiently comparable to Divac's to suggest that the additional training was critical to the lesion effects seen and that the negative findings reported here may be useful.

\section{METHODS}

\section{Subjects}

Twenty-two male hooded rats (Blue Spruce Farms, Inc., Altamont, N.Y.), 90 days old, were used. Based on preoperative spatial reversal performance, matched groups were selected to receive either parietal $(n=6)$, MF $(n=6)$ or FP $(n=6)$ lesions or sham operations $(n=5)$. These groups were subsequently given further spatial reversal training.

\section{Surgery and histological analysis}

Following an intraperitoneal injection of Equithesin (Jensen-Salsbery Laboratories; $0.25 \mathrm{cc} / 100 \mathrm{~g}$ of body weight), the dorsal hair on the cranium was clipped and a midline incision of the scalp was made. The parietal, medial frontal, or frontopolar cortices were exposed by drilling the skull with a trephine. Locating a trephine with an outside diameter of 3.5 $\mathrm{mm}$, bilaterally $1 \mathrm{~mm}$ from the saggital suture and $1 \mathrm{~mm}$ posterior to the coronal suture and suctioning the area beneath the opening yielded the desired size and position for the parietal lesions. The same trephine was used bilaterally at positions about $1 \mathrm{~mm}$ from the saggital suture and $1 \mathrm{~mm}$ anterior to the coronal suture to achieve the desired FP lesions. A trephine with an outside diameter of $5.5 \mathrm{~mm}$ was centered on the midline with the posterior edge even with the coronal structure to make the 


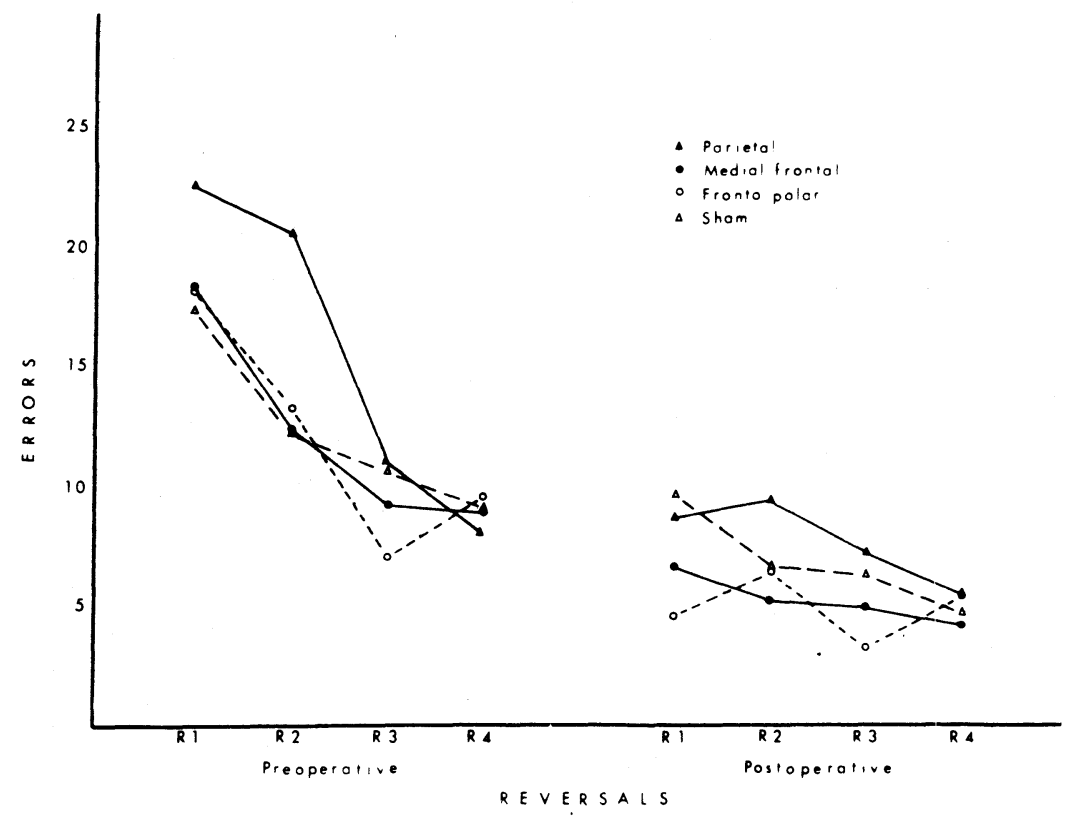

Figure 1. Mean errors of the frontopolar, medial frontal, parietal and sham operated groups on the preoperative and postoperative reversal problems. opening for the MF lesions. Following aspiration of the neocortex, the scalp was sutured and powdered with a tetracycline medication. Sham operations consisted of proceeding to the point of drilling through the skull. At this point, the incision was sutured, the cranium dusted with tetracycline and the animal was returned to his home cage to recover.

\section{Anatomical}

At the end of the experiment, the rats were sacrificed with an overdose of Equithesin. After surgical anesthesia was reached, a thoracic incision was made, the heart was exposed and intracardial perfusion of $50 \mathrm{cc}$ of $0.85 \%$ saline followed by $50 \mathrm{cc}$ of $10 \%$ formalin was done. The brain was then placed in $10 \%$ formalin for a minimum of 2 days, after which it was photographed on continuous tone Polaroid projection film. The brain was then embedded in celloidin, sectioned at 40 micrometers, and stained (every fifth section) with thionin or by the Weil method for myelin, alternately. The projection slide of the whole brain was focused on a Lashley brain diagram, adjusted so the image and the diagram were in a 1:1 relationship, and the lesion boundaries were traced on the diagram. Similarly, the images of a representative medial frontal lesion in a coronal section were drawn on tracings of the rostral 14 diagrams from Konig and Klippel (1963). Percentages of cerebral cortical destruction were determined for the FP and parietal lesions using the dot grid method (Thomas \& Peacock, 1965). MF lesion percentages were not computed, because Lashley did not include the medial cortical surfaces in his denominator, and in the context of the larger work of which the present experiment was a part, comparison with Lashley's (1929) percentages was desired.

\section{Apparatus and procedure}

The rats were deprived of food for $48 \mathrm{~h}$ and were trained initially in a straight runway $(10.2 \times 10.2 \times 304.8 \mathrm{~cm}$, painted gray and with a wire mesh top). Sliding doors formed a startbox and a goalbox $30.5 \mathrm{~cm}$ from either end. A rat first learned to eat two Noyes food pellets $(.045 \mathrm{~g})$ in the goalbox on each of five daily trials. Then he was trained to go from the startbox to the goalbox in five trials daily for two food pellets until he traversed the runway within $10 \mathrm{sec}$ on three consecutive trials. Subsequent to this, the rat was trained to eat food pellets in both goal boxes of the spatial reversal apparatus. Finally, in pretraining, each animal was given one trial in the spatial reversal apparatus with the opportunity to find the food reinforcers in either goalbox. The goalbox opposite the one chosen by the animal in this preliminary trial was selected as the initial site to be reinforced when training began. Other than the pellet reinforcers, the animais were fed a maintenance ration (12-15 g) of Purina Rat Chow immediately following each daily session.

The spatial reversal apparatus was constructed of wood, painted gray and covered with wire mesh according to the plan shown by Divac (1971). The essential features of this apparatus are a startbox $(11.5 \mathrm{~cm}$ wide $\times 27 \mathrm{~cm}$ long $\times 14 \mathrm{~cm}$ high $)$ opening into an area where, at a distance of $38 \mathrm{~cm}$, a choice point between two parallel alleys $(17.8 \mathrm{~cm}$ wide $x 50.0 \mathrm{~cm}$ long $x 14 \mathrm{~cm} \mathrm{high)} \mathrm{is} \mathrm{reached.} \mathrm{A} \mathrm{clear} \mathrm{Plexiglas} \mathrm{guillotine} \mathrm{door} \mathrm{may}$ be operated at the startbox. Each goalbox may be enclosed by a guillotine door located $32 \mathrm{~cm}$ from the end of either alley.

Upon the rat's exit from the startbox, its door was closed. When the rat entered the correct alley, a gray guillotine door was closed behind him. The rat found two food pellets in a small (4.4 $\mathrm{cm}$ dia) aluminum dish at the end of this alley. When the rat entered the incorrect alley, a black and white striped door was closed behind him and he found a black and white striped block of wood instead of the food dish, and a 30-w light immediately above the goalboxes (1650 Visual Stimulator, Lafayette Instrument Co.) and a $58 \mathrm{DB}$ (re $0.0002 \mathrm{dynes} / \mathrm{cm}^{2}$ measured from the center of the enclosed portion of the alley) buzzing sound were presented.

The rats were given 10 noncorrection trials per day with intertrial intervals of approximately $10 \mathrm{sec}$ until eight correct responses in one session were made. Following criterion performance and beginning the next session, the alley specified as correct was reversed. Preoperative training consisted of the initial training and four subsequent reversals. Surgery was performed the day after completion of the fourth reversal. Ten days postoperatively, retraining to the same criterion began. The alley other than that which had been correct on the fourth preoperative reversal was designated correct. This initial postoperative training was followed by four reversals. 


\section{RESULTS AND DISCUSSION}

A two-factor analysis of variance with repeated measures on one factor and appropriate adjustments for unequal groups revealed no significant differences in errors among the groups on either the preoperative or the postoperative reversals (Figure 1). There was a significant reduction in mean errors postoperatively compared to mean errors preoperatively $[\mathrm{F}(3 / 18)=55.35, \mathrm{p}<.01]$.

The tracings of the lesions on Lashley brain diagrams may be seen in Figure 2. Figure 3 shows a representative MF lesion. Examination of the coronal sections revealed slight damage to the corpus callosum in two animals with MF lesions and in five animals with parietal lesions.

Our failure to confirm Divac's (1971) report of spatial reversal deficits following MF lesions appears to be due to differences in the preoperative experience of the control groups there and here. Several observations support this interpretation, but first, it should be recalled that his rats were given either 64 or 104 reversals prior to being lesioned, whereas our rats received only four reversals prior to surgery. (a) Despite the greater preoperative training of Divac's rats, his MF lesioned animals and our MF lesioned animals performed similarly. Divac's MF group which received 64 reversals preoperatively had a mean of 6.3 errors postoperatively; his MF group which received 104 reversals preoperatively had a mean of 5.2 errors postoperatively. Our MF group also had a mean of 5.2 errors postoperatively. Statistical comparison (Mann-Whitney $\mathrm{U})$ of his combined MF groups' postoperative data with our MF group's postoperative data showed no significant difference. (b) Inspection of Figure 1 suggests that neither the surgery nor the allotted time for recovery appears to have affected postoperative reversal performance. The acquisition curves appear to have resumed postoperatively from the approximate point of preoperative terminal performance. (c) Statistical comparison of Divac's combined FP lesion + nonoperated control groups (mean errors $=3.2$ ) and our combined FP lesion + sham-operated control groups (mean errors $=6.2$ ) showed his control groups to have significantly fewer errors than our groups postoperatively, Mann-Whitney $U=14 ; n_{1}=10$, $n_{2}=10 ; p<.02$.

Thus, while Divac's MF lesion group showed a postoperative deficit compared to his control groups, his MF group does not seem to have differed from our MF group. Divac's demonstration of a spatial reversal deficit following MF lesions appears to have been dependent upon the effects of additional preoperative reversal training given his control group. This interpretation of the findings does not question the validity or usefulness of Divac's relults. To the contrary, it points to the subtle influence of MF lesions on spatial reversal performance.

Nonneman, Voigt, and Kolb (1974) confirmed Divac's (1971) finding of spatial reversal deficits following MF lesions. Their animals received 10 preoperative and 5 postoperative reversals. While they did not specify the mean errors, interpretation of their graph suggests that the controls had reached asymptotic levels of performance with the 10 preoperative reversals.
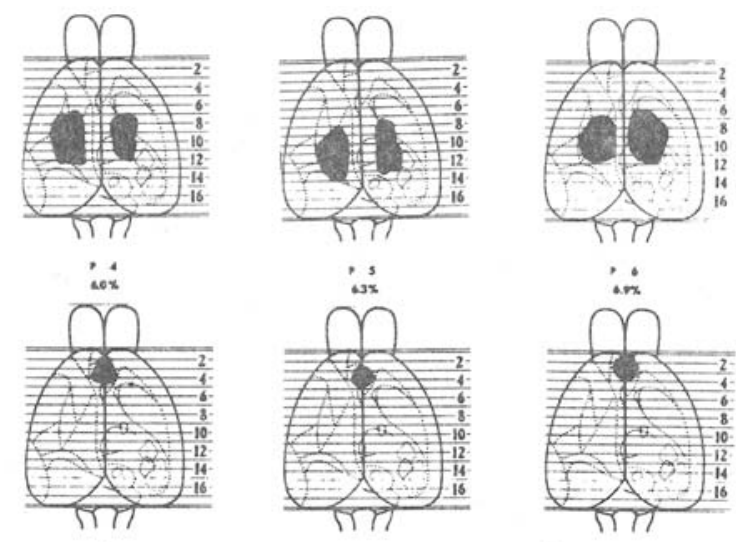

$\therefore$

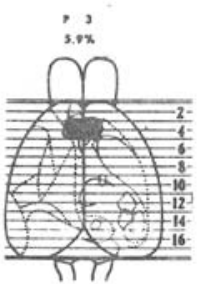

mo 3
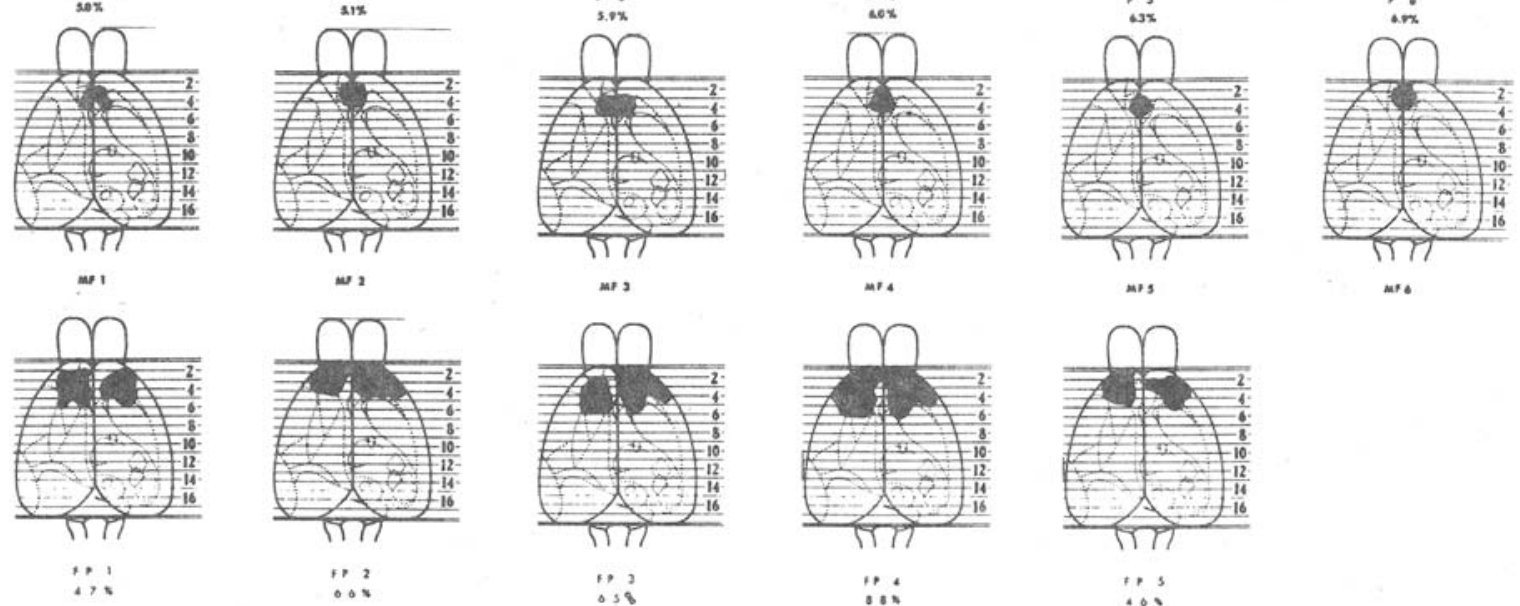

$\therefore s$

Figure 2. Lashley brain diagrams illustrating the extent of dorsal neocortical damage in the frontopolar (FP), medial frontal (MF), and parietal (P) neocortical lesion groups. 

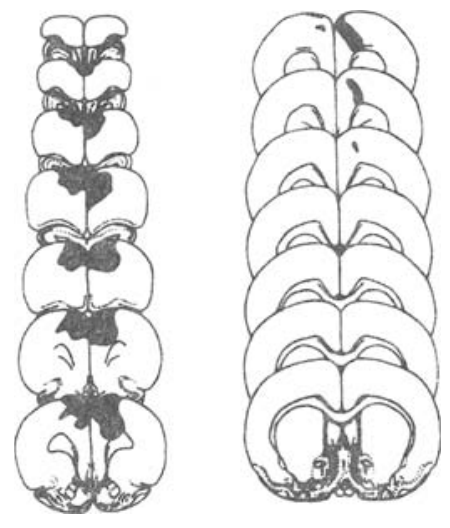

Figure 3. A representative medial frontal lesion superimposed on tracings of the rostral 14 sections from Konig and Klippel, 1963.

Therefore, while four preoperative reversals may be insufficient, 10 reversals, rather than 64 or 104, preoperatively may be sufficient to demonstrate the spatial reversal effect following medial frontal lesions. Hoeever, it must be noted that the MF lesions shown by Nonneman et al. appear to be larger than those shown by Divac or us. The lesions shown in Divac's study appeared to us to be comparable to the lesions in the present work. Finally, it may be noted that Kolb, Nonneman, and Singh (1974) have shown that rats trained on spatial reversal problems after having received
MF lesions failed to show interproblem improvement, whereas controls and rats with orbital frontal lesions did show significant improvement.

\section{REFERENCES}

Clark, W E LeGros. An experimental study of thalamic connections in the rat. Philosophical transactions, Royal Society of London, 1932, 222B, 1-28.

Diamond, I. T. \& Hall, W. C. Evolution of neocortex. Science, $1969,164,251-262$.

Divac I Frontal lobe system and spatial reversal in the rat. Neuropsy chologia, 1971, 9, 175-183.

Gurdjian, E. S. The diencephalon of the albino rat. Journal of Cornparative Neurology, 1927, 43, 1-114.

Kolb, B., Nonneman, A. J., \& Singh, R. K. Double dissociation of spatial impairment and perseveration following selective prefrontal lesions in rats. Journal of Comparative and prefrontal lesions in rats. Journal of

Konig, J. F. R., \& Klippel, R. A. The rat brain. Baltimore: Williams and Wilkens, 1963.

Lashley, K. S. Brain mechanisms and intelligence. Chicago: University of Chicago Press, 1929.

Lashley, K. S. Thalamo-cortical connections of the rat's brain. Journal of Comparative Neurology, 1941, 75, 67-121.

Leonard, C. M. The prefrontal cortex of the rat. I. Cortical projections of the mediodorsal nucleus. II. Efferent projections of the mediodorsal nucleus.
connections. Brain Research, 1969, 12, 321-343.

Nonneman, A. J., Voigt, J., \& Kolb, B. E. Comparisons of behavioral effects of hippocampal and prefrontal cortex lesions in the rat. Journal of Comparative and Pyysiological Psychology, 1974, 87, 249-260.

Thomas, R. K. Mass function and equipotentiality: A reanalysis of Lashley's retention data. Psychological Reports, 1970, 27, 899-902.

Thomas, R. K. \& Peacock, L. J. A method for measuring brain lesions. Psychonomic Science. 1965. 3. 184.

Thomas, R. K., and Weir, V. K. The effect of lesions in the frontal or posterior association corte $x$ of rats on maze III. Physiological Psy chology, in press.

(Received February 3, 1975.) 